\title{
Development of a nomogram incorporating serum C-reactive protein level to predict overall survival of patients with advanced urothelial carcinoma and its evaluation by decision curve analysis
}

\author{
J Ishioka', K Saito*,', M Sakura', M Yokoyama', Y Matsuoka', N Numao', F Koga', H Masuda', Y Fujii', \\ S Kawakami' and K Kihara' \\ 'Department of Urology, Tokyo Medical and Dental University, I-5-45 Yushima, Bunkyo-ku, Tokyo I I 3-85 I 0, Japan
}

\begin{abstract}
BACKGROUND: The purpose of this study is to investigate the prognostic impact of C-reactive protein (CRP) on patients with advanced urothelial carcinoma and to develop a novel nomogram predicting survival.

METHODS: A total of 223 consecutive patients were treated at Tokyo Medical and Dental Hospital. A nomogram incorporating $\vee$ was developed based on the result of a Cox proportional hazards model. Its efficacy and clinical usefulness was evaluated by concordance index (c-index) and decision curve analysis.

RESULTS: Of the 223 patients, I 84 (83\%) died of cancer. Median follow-up periods of patients who died and those who remained alive were 5 and II months, respectively. We developed a novel nomogram incorporating Eastern Cooperative Oncology Group Performance Status, presence of visceral metastasis, haemoglobin and age. The c-index of the nomogram predicting survival probability 6 and 12 months after diagnosis was 0.788 and 0.765 , respectively. Decision curve analyses revealed that the novel nomogram incorporating CRP had a superior net benefit than that without CRP for most of the examined probabilities.

CONCLUSION: We demonstrated the prognostic impact of CRP that improved the predictive accuracy of a nomogram for survival probability in patients with advanced urothelial carcinoma.

British Journal of Cancer (2012) I07, I031-1036. doi:I0.1038/bjc.2012.254 www.bjcancer.com

Published online 23 August 2012

(C) 2012 Cancer Research UK
\end{abstract}

Keywords: C-reactive protein; advanced urothelial carcinoma; biomarker; survival prediction; nomogram; decision curve analysis

The prognosis of locally advanced or metastatic urothelial carcinoma (UC) is poor despite recent advances in the systemic chemotherapy regimen. Although previous reports have demonstrated an initial good response to chemotherapy, numerous patients were unable to achieve a reasonable survival period (von der Maase et al, 2005; Malmström, 2008). Even in those patients, the precise pretreatment evaluation of the prognosis enabled clinicians to offer better therapeutic strategy, such as clinical trials or symptom control (Bellmunt et al, 2010).

Several prognostic factors for overall survival of advanced UC have been identified using clinical trial cohorts. In previous reports, variables such as performance status (PS), presence of visceral metastasis, and anaemia have been reported to predict prognosis. Some of the risk stratification models using these factors have been utilised to standardise patient risk in clinical trials (Bajorin et al, 1999; von der Maase et al, 2005; Bellmunt et al, 2010).

Recently, the presence of a systemic inflammatory response represented by C-reactive protein (CRP) has been demonstrated to be associated with poor outcome in various advanced cancers (Roxburgh and McMillan, 2010). We and other investigators demonstrated the prognostic impact of CRP in urologic cancers (Saito et al, 2007; Yoshida et al, 2008). An elevated CRP

*Correspondence: Dr K Saito; E-mail: kz-saito@tmd.ac.jp

Received I February 2012; revised I May 2012; accepted II May 20 I2; published online 23 August 2012 concentration predicts poor prognosis. C-reactive protein has been incorporated into prognostic models and has improved the predictive accuracy for bladder UC, the so-called TNR-C score, and renal cell carcinoma, the so-called TNM-C score (Iimura et al, 2009; Gakis et al, 2011). As measurement of CRP has been established and has become widely available, CRP can generally be tested in current clinical practice. Therefore, we hypothesise that CRP could also be considered a strong prognostic factor to improve the predictive accuracy of a prognostic model for advanced UC.

The purpose of this study was to investigate the impact of CRP on advanced UC and to develop a prognostic nomogram that incorporates CRP using a cohort of patients with advanced UC who received heterogeneous treatments of cisplatin-based chemotherapies, radiotherapy, and/or best supportive care at a single institution.

\section{MATERIALS AND METHODS}

\section{Patients}

Between January 1995 and April 2010, a total of 232 consecutive patients with advanced UC were treated at Tokyo Medical and Dental University Hospital. The diagnosis of UC was confirmed by histological examinations and the extent of the disease was evaluated by computed tomography, magnetic resonance imaging, 
and/or bone scintigraphy. The patients include those with locally advanced (cT4 unresectable disease) or distant metastases (lymph node and/or visceral metastases). Patients with missing data at diagnosis (CRP, $n=3$; alkaline phosphatase (ALP), $n=2$; haemoglobin $(\mathrm{Hb}), n=4)$ were excluded. The remaining 223 patients constituted the current study cohort. At the time of diagnosis, blood count, creatinine, ALP, aspartate aminotransferase, lactate dehydrogenase (LDH), albumin, and CRP were evaluated. The total number of patients treated by cisplatin-based chemotherapies, radiotherapy, and metastasectomy were $101(45 \%), 83(37 \%)$, and 7 (3\%), respectively; however, $85(38 \%)$ patients were treated solely with best supportive care owing to their poor general condition or their choice. In all patients, survival and final status at last visit were determined from the medical records. All study participants provided informed consent, and the study design was approved by an ethics review board. The overall survival period was estimated from the duration between the date of diagnosis and death or last follow-up.

\section{Variables}

Factors analysed for association as overall survival included age, gender (male vs female), Eastern Cooperative Oncology Group Performance Status (ECOG PS) $(<2$ vs $\geqslant 2)$, white blood cell count, visceral metastases (including any non-lymph node metastasis), lymph node metastasis, hydronephrosis, Hb, creatinine, ALP, LDH, corrected calcium, albumin, CRP, history of prior definitive therapies, and primary site (bladder $v s$ non-bladder). Corrected calcium, which was regarded as normal up to $10 \mathrm{mg} \mathrm{dl}^{-1}$, was calculated with the formula of total calcium 0.707 (albumin - 3.4). Serum CRP level was quantified by latex agglutination immunoassay with a CRP-L kit (Mitsubishi Kagaku Iatron Co. Ltd., Tokyo, Japan) from 1995 to 2005 and with a Nanopia CRP kit (Daiichi Pure Chemicals Co. Ltd., Tokyo, Japan) from 2006 to 2010, as reported antecedently (Tatokoro et al, 2008). The values of all parameters used in this analysis were measured when patients were diagnosed as having advanced UC. Patients were free from infectious disease and collagen disease, both of which affect serum CRP levels. When necessary, log transformations of continuous variables were used to reduce the skew of their distributions.

\section{Statistical methods}

The immediate overall survival estimates after diagnosis of advanced UC were calculated with the Kaplan-Meier method. The multivariate model with backward manual selection of statistically significant variables was carried out $(P<0.05)$. Nomograms predicting the 6 - or 12 -month survival probability were developed based on the result of the Cox proportional hazards model.

The discriminative ability of the final multivariate model was expressed by the Harrell's concordance index (c-index), which lays out an alteration of the area under the curve method when censored observations are present, as reported previously (Harrell et al, 1982). Internal bootstrap validation, bias-corrected $95 \%$ confidence intervals for hazard ratios in the final model, and bootstrap optimism corrected c-index were calculated using 1000 re-samples (Chen and George, 1985).

To evaluate the net benefit derived from the final multivariate models with or without CRP, we relied on decision curve analysis, as described by Vickers et al and previously applied by the authors and their colleagues in the context of prostate repeat biopsy (Vickers and Elkin, 2006; Vickers et al, 2008; Lughezzani et al, 2010; Vickers and Cronin, 2010; Sakura et al, 2011). Decision curve analysis examines the theoretical relationship between the threshold survival probability at 6 and 12 months of advanced UC and the relative value of false-positive and false-negative results to determine the value of a prediction model (Vickers and Elkin, 2006; Vickers et al, 2008; Vickers and Cronin, 2010).

All analyses were performed using R.2.11.0 (The R Foundation for Statistical Computing, 2010). The $\mathrm{R}$ code for decision curve analysis can be found at http://www.decisioncurveanalysis.org along with tutorials on using the code.

\section{RESULTS}

\section{Patient demographics and outcome}

The demographics of the patients are listed in Table 1 . Of the 223 patients, $184(83 \%)$ died of cancer and the remaining $39(17 \%)$ were censored (alive with disease $36(16 \%)$, no evidence of disease $3(1 \%))$. Median follow-up periods of patients who died of cancer and those who are alive were 5 and 11 months, respectively. The 6- and 12-month survival rates were $48 \%$ and $30 \%$, respectively (Figure 1). The median CRP level was $15.0 \mathrm{mgl}^{-1}$ $\left(0.1-207.0 \mathrm{mgl}^{-1}\right)$.

\section{Prognostic factors for overall survival}

Univariate analysis revealed that age, ECOG PS $(\geqslant 2), \mathrm{Hb}$ $\left(<10 \mathrm{~g} \mathrm{dl}^{-1}\right)$, albumin $\left(<3 \mathrm{~g} \mathrm{dl}^{-1}\right)$, CRP (continuous), ALP (continuous), LDH (continuous), visceral metastases, hydronephrosis, primary organ, corrected calcium, and lymph node metastasis were significant factors in the prediction of overall survival (Table 2). Multivariate analysis revealed that age (continuous), ECOG PS $(\geqslant 2), \mathrm{Hb}\left(<10 \mathrm{~g} \mathrm{dl}^{-1}\right)$, CRP (continuous), LDH (continuous), visceral metastases, and lymph node metastases were independent prognostic factors for overall survival. By using these factors, we developed a prognostic model for predicting 6- and 12-month survival probability. The bootstrap resampling technique confirmed the bootstrap-calculated $95 \%$ confidence interval for each variable of the full model and the model without CRP (Table 3).

\section{C-reactive protein status and overall survival}

C-reactive protein was a continuously significant prognostic factor. As the level of CRP increases, the overall survival period tends to be shorter. When patients were stratified according to pretreatment CRP level (cut-off values are 5, $15 \mathrm{mgl}^{-1}$ ), overall survival curves were statistically significant according to pretreatment CRP levels with median survival periods of 16 months $\left(\mathrm{CRP}<5 \mathrm{mgl}^{-1}\right), 7$ months $\left(5 \leqslant \mathrm{CRP}<15 \mathrm{mgl}^{-1}\right)$, and 3 months $\left(15 \mathrm{mgl}^{-1} \leqslant \mathrm{CRP}\right)(P<0.001)$ (Figure 2$)$.

\section{Development of nomograms}

Two nomograms for the prediction of survival at 6 and 12 months after diagnosis were developed. First, we constructed a nomogram that consisted of age, ECOG PS $(<2$ vs $\geqslant 2), \mathrm{Hb}(<10 v s$ $\left.>10 \mathrm{~g} \mathrm{dl}^{-1}\right), \mathrm{LDH}$, visceral metastases (present $v s$ absent), and lymph node metastases (present $v s$ absent). Second, a nomogram combined with additional parameters of CRP as continuous variables was constructed (Figure 3). C-reactive protein was a significant factor for the nomogram and adding CRP to the nomogram improved the c-index of 0.788 by 0.023 .

The calibration plots for internally validated censored data indicated a well-balanced and evenly distributed prediction. The decision curve analyses indicated that the full model resulted in a higher net benefit for most of the predicted survival probabilities (Figure 4). For example, 6 months after diagnosis, if a survival probability at the point of $50 \%$ is used as a threshold, the net benefit of the full model is 0.32 , which is superior to 0.24 for the model without CRP (Figure 5). The prognostic model with CRP is not only accurate but also has practical superiority over that 
Table I Patient demographics and clinical characteristics at diagnosis

\begin{tabular}{|c|c|}
\hline Clinical characteristics & No. of patients (\%) \\
\hline \multicolumn{2}{|l|}{ Gender } \\
\hline Male & $162(73)$ \\
\hline Female & $61(27)$ \\
\hline \multicolumn{2}{|l|}{ Age, years } \\
\hline Median & 71 \\
\hline Quartiles & $66-77$ \\
\hline \multicolumn{2}{|l|}{ ECOG Performance status } \\
\hline 0 & $43(19)$ \\
\hline । & $74(33)$ \\
\hline$\geqslant 2$ & $106(48)$ \\
\hline Hydronephrosis & $81(37)$ \\
\hline \multicolumn{2}{|l|}{ Primary site } \\
\hline Bladder & $190(59)$ \\
\hline Non-bladder & $91(41)$ \\
\hline Prior definitive therapy & $168(75)$ \\
\hline Lymph node metastasis & $129(42)$ \\
\hline Any visceral metastasis & 117 (47) \\
\hline Liver & $26(12)$ \\
\hline Bone & $50(23)$ \\
\hline Lung & $55(25)$ \\
\hline \multicolumn{2}{|l|}{ WBC } \\
\hline Median & 6900 \\
\hline Quartiles & $5400-9000$ \\
\hline \multicolumn{2}{|l|}{ Haemoglobin, $g d^{-1}$} \\
\hline$\geqslant 10$ & $162(73)$ \\
\hline$<10$ & $61(27)$ \\
\hline \multicolumn{2}{|l|}{ Creatinine, $m g d^{-1}$} \\
\hline Median & 1.09 \\
\hline Quartiles & $0.8-1.37$ \\
\hline \multicolumn{2}{|l|}{ Albumin, $g d^{-1}$} \\
\hline Median & 3.60 \\
\hline Quartiles & $3.10-4.00$ \\
\hline \multicolumn{2}{|l|}{$A L P, U I^{-1}$} \\
\hline Median & 257.0 \\
\hline Quartiles & $208.0-348.2$ \\
\hline \multicolumn{2}{|l|}{$\mathrm{LDH}, \cup \mathrm{I}^{-1}$} \\
\hline Median & 221.0 \\
\hline Quartiles & $|80.0 .0-3| 1.0$ \\
\hline \multicolumn{2}{|l|}{ Corrected calcium, $m g \mathrm{dl}^{-1}$} \\
\hline Median & 9.188 \\
\hline Quartiles & $8.922-9.47 \mid$ \\
\hline \multicolumn{2}{|l|}{ CRP, $m g l^{-1}$} \\
\hline Median & 15.0 \\
\hline Quartiles & $2.0-54.0$ \\
\hline
\end{tabular}

Abbreviations: $\mathrm{ALP}=$ alkaline phosphatase; $\mathrm{LDH}=$ lactate dehydrogenase; $\mathrm{CRP}=$ C-reactive protein; $\mathrm{ECOG}=$ Eastern Cooperative Oncology Group; $\mathrm{WBC}=$ white blood cell.

without CRP, because the curve of its prediction is plotted above the curve without CRP.

\section{DISCUSSION}

In this study, we demonstrated the first nomogram including CRP that predicts the prognosis of patients with advanced UC in real-world cohorts. CRP was a significant prognostic factor in

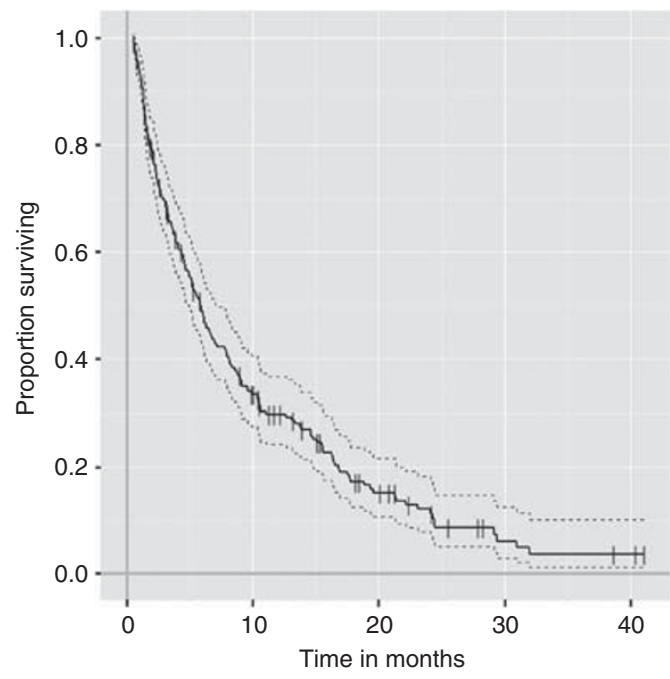

Figure I Kaplan-Meier estimates of overall survival.

Table 2 Univariate analysis for overall survival

\begin{tabular}{|c|c|c|c|}
\hline & HR & $95 \% \mathrm{Cl}$ & $P$ \\
\hline Gender: male & 1.007 & $0.966-1.398$ & 0.966 \\
\hline Age & 1.024 & $1.007-1.04$ & 0.004 \\
\hline \multicolumn{4}{|l|}{ Poor performance status } \\
\hline$(E C O G P S \geqslant 2)$ & 4.094 & $3.007-5.575$ & $<0.001$ \\
\hline Haemoglobin $<10 \mathrm{~g} \mathrm{dl}^{-1}$ & 2.187 & $1.585-3.018$ & $<0.001$ \\
\hline $\log (W B C)$ & 2.046 & $1.496-2.796$ & $<0.001$ \\
\hline Creatinine & 1.081 & $1.000-1.170$ & 0.050 \\
\hline Albumin: $<3 \mathrm{gdl}^{-1}$ & 2.337 & $1.597-3.420$ & $<0.001$ \\
\hline $\log (C R P)$ & 2.362 & $1.930-2.890$ & $<0.001$ \\
\hline $\log (A L P)$ & 3.680 & $2.045-6.620$ & $<0.001$ \\
\hline $\log (\mathrm{LDH})$ & 4.870 & $2.682-8.84$ & $<0.001$ \\
\hline Visceral metastasis & 1.980 & $1.474-2.659$ & $<0.001$ \\
\hline Hydronephrosis & 1.476 & $1.095-1.990$ & 0.011 \\
\hline \multicolumn{4}{|l|}{ Primary organ } \\
\hline Upper urinary tract & 0.681 & $0.503-0.921$ & 0.013 \\
\hline Corrected calcium & 1.319 & $1.098-1.586$ & 0.003 \\
\hline Lymph node metastasis & 0.711 & $0.528-0.956$ & 0.024 \\
\hline Prior definitive therapy & 0.817 & $0.585-1.14 \mid$ & 0.236 \\
\hline
\end{tabular}

Abbreviations: $\mathrm{ALP}=$ alkaline phosphatase; $\mathrm{Cl}=$ confidence interval; $\mathrm{CRP}=$ C-reactive protein; ECOG PS = Eastern Cooperative Oncology Group Performance Status; $H R=$ hazard ratio; $L D H=$ lactate dehydrogenase; $W B C=$ white blood cell.

patients with advanced UC. Incorporated into a prognostic algorithm such as a nomogram, CRP improved the predictive accuracy.

Abnormalities in relation to inflammation comprise a large amount of disorders, and one of cancer's aetiological origins is believed to reside in the inflammatory processes. As the association between inflammation and cancer prognosis becomes better understood, an acute phase reactant has been demonstrated as significant in terms of predicting outcomes (López-Novoa and Nieto, 2009). C-reactive protein is a representative acute phase reactant that is widely used to evaluate systemic inflammation. C-reactive protein can be measured with a reliable assay kit that is affordable worldwide (Karakiewicz et al, 2007; Iimura et al, 2009; Gakis et al, 2011; Saito and Kihara, 2011).

As previously reported for other advanced cancers, the presence of an elevated CRP level that reflects the presence of systemic 
Table 3 Multivariate analysis for overall survival

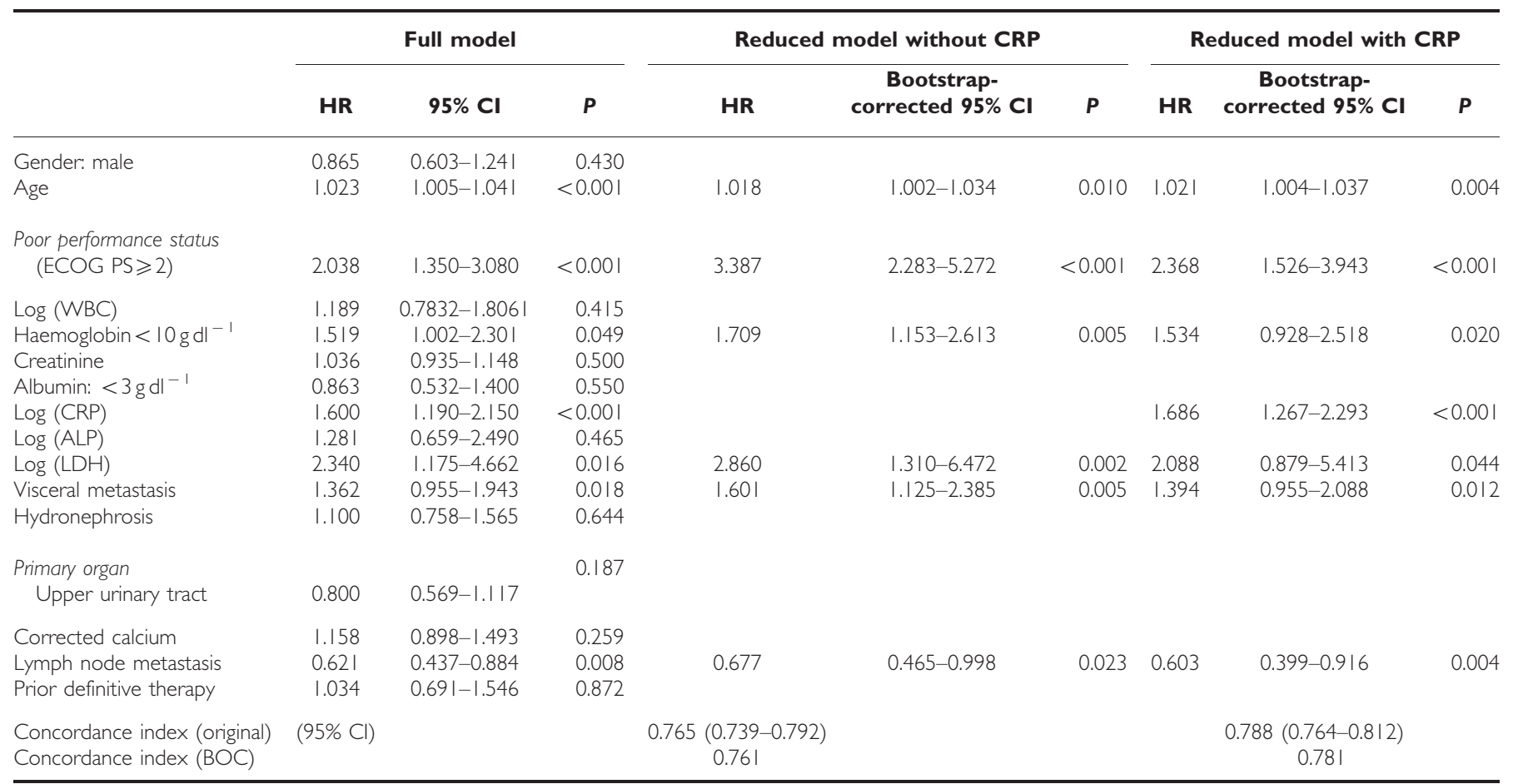

Abbreviations: $\mathrm{ALP}=$ alkaline phosphatase; $\mathrm{BOC}=$ Bootstrap optimism corrected; $\mathrm{CRP}=\mathrm{C}$-reactive protein; $\mathrm{Cl}=$ confidence interval; $\mathrm{ECOG} \mathrm{PS}=\mathrm{Eastern} \mathrm{Cooperative}$ Oncology Group Performance Status; HR = hazard ratio; LDH = lactate dehydrogenase; WBC = white blood cell.

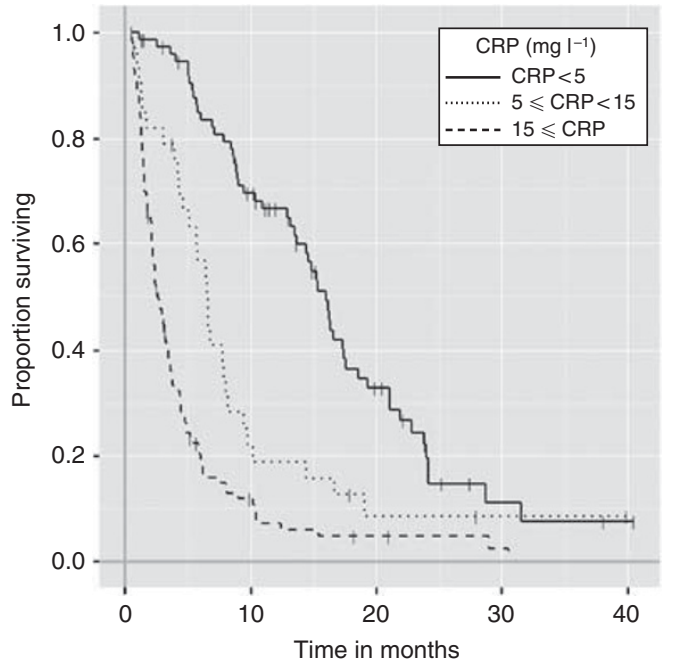

Figure 2 Kaplan-Meier estimates of overall survival are calculated; the median survival periods of patients are 16.0 months $\left(C R P<5 \mathrm{mg} \mathrm{I}^{-1}\right), 6.5$ months $\left(5 \mathrm{mgl}^{-1} \leqslant \mathrm{CRP}<|0 \mathrm{mg}|^{-1}\right), \quad 3.8$ months $\left(|0 \mathrm{mg}|^{-1} \leqslant C R P\right.$ $\left.<30 \mathrm{mgl}^{-1}\right)$, and 2.6 months (30 $\mathrm{mgl}^{-1} \leqslant \mathrm{CRP}$ ).

inflammatory response was linked to poor prognosis in advanced UC (Saito et al, 2007; Yoshida et al, 2008; Roxburgh and McMillan, 2010). In many previous papers, however, the significance of CRP as a prognostic factor was evaluated in a dichotomised fashion. The current study revealed that the significance of CRP can also be demonstrated as a continuous variable that can have an important role in a nomogram of the survival of patients with advanced UC.

Although certain prognostic factors have been identified in clinical trial cohorts, it remains unknown if the factors that are applicable to the daily practical cohort differ from those of the clinical trial cohort (Bajorin et al, 1999; von der Maase et al, 2005; Jessen et al, 2009; Bellmunt et al, 2010). The cohort of the current study was composed of a heterogeneous patient group, in which some of the patients did not receive systemic therapies. Approximately $40 \%$ of the patients were treated solely by best supportive care. Our populations and analytic approach, however, may reflect the complexity and diversity of actual clinical practice. We believe that our novel nomogram could be utilised in daily clinical practice for advanced UC.

A number of simple inflammation-based prognostic scores such as the TNM-C score, the Glasgow Prognostic Score, and the Neutrophil Lymphocyte Ratio have already been proposed (Ramsey et al, 2007). However, the continuous probabilities for survival rate calculated with a nomogram provide advanced UC patients with more helpful information, because individualised survival probabilities based on an individual's disease characteristics can be obtained. Although risk grouping is a tool that is easy to understand and use, it assumes that all patients within a risk group are equal, and results in information loss (spectrum bias). Moreover, using an electronic version of a nomogram mitigates the issue of complexity (Shariat et al, 2008; Ingram and Kattan, 2011).

The usefulness of any marker should be demonstrated by the improvement in the predictive accuracy of a multivariate model with established factors as well as its own prognostic significance. C-reactive protein could meet the criteria that the predictive accuracy expressed by the c-index was improved by adding CRP to a multivariable model for advanced UC. Furthermore, the decision curve analysis plots depicted the benefit of using the model with CRP. The prognostic nomogram including CRP exhibited improved benefit over the entire range of threshold probabilities.

Interestingly, the present nomogram showed a similar c-index value for patients with presumably locally confined bladder cancer who underwent curative intended surgery, as shown by the TNR-C score. This demonstrates that serum CRP serves as a prognostic marker in both metastatic settings as well as in locally confined settings (Gakis et al, 2011). 


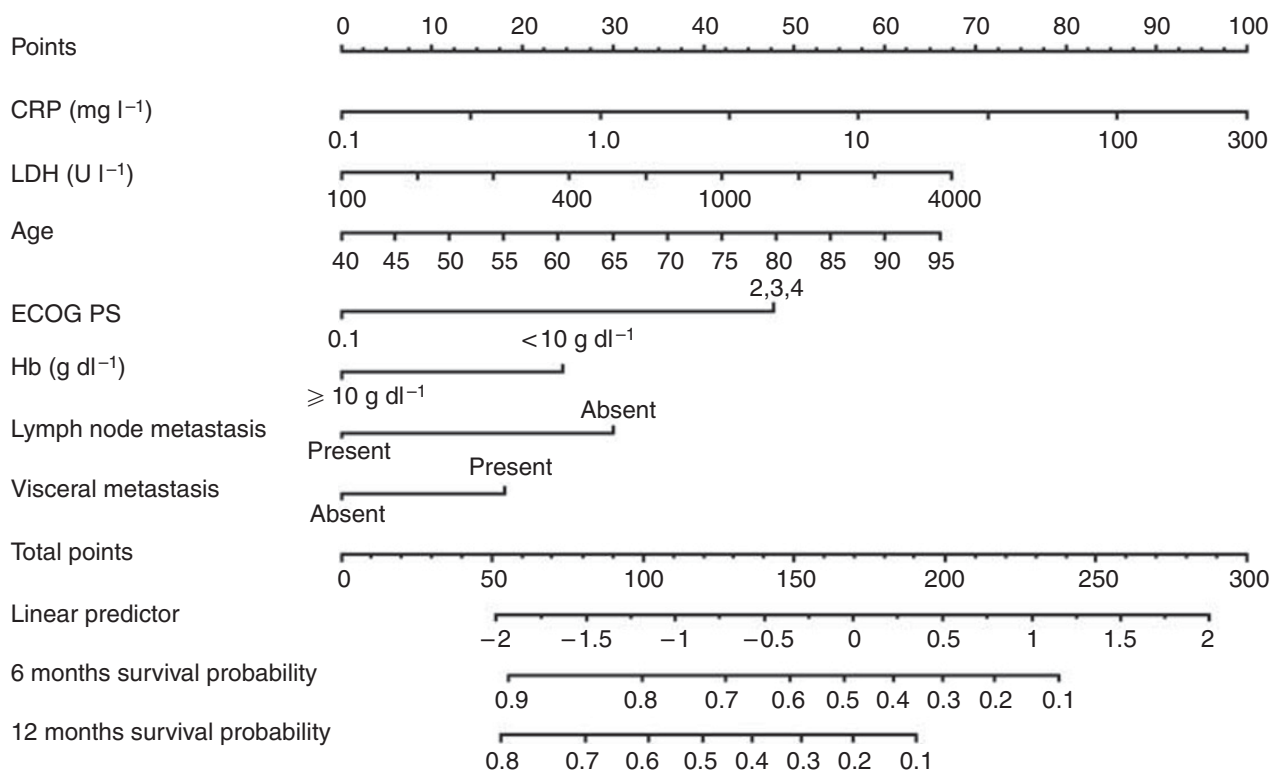

Figure 3 Nomogram depicting estimates of 6-and 12-month overall survival. To obtain the predicted probability 6 and 12 months after diagnosis of advanced UC, locate the patient values on each axis. Draw a vertical line upward to the 'Points' axis to determine the points of the variable. Sum the points for all variables and locate the sum on the 'Total points' axis. Draw a vertical line down to the 'Probability of 6-month survival from diagnosis' axis to find the patient's probability of survival at 6 months. Abbreviations: $C R P=C$-reactive protein; $L D H=$ Lactate dehydrogenase; $E C O G$ PS $=$ Eastern Cooperative Oncology Group Performance Status; $\mathrm{Hb}=$ Haemoglobin.

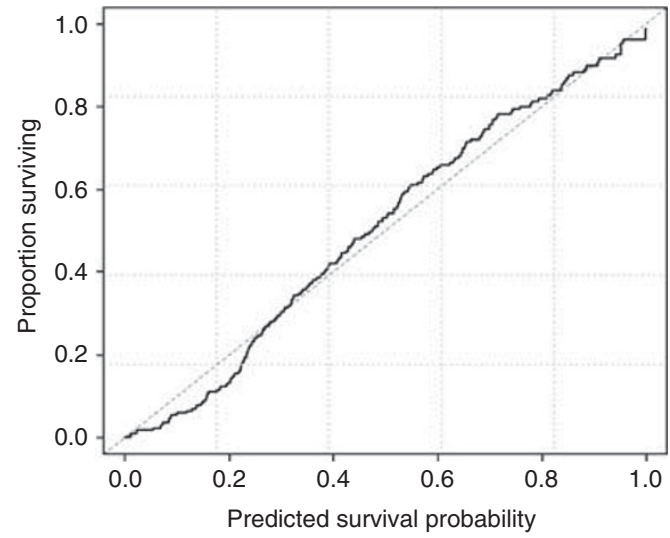

Figure 4 Comparison between nomogram-predicted probability of overall survival ( $x$-axis) and the actual fraction surviving ( $y$-axis) within the internal validation cohort.

As this retrospective study population is relatively small and is not uniform in terms of treatment modality, further studies are needed to confirm the current results. Our cohort included $30 \%$ of patients who did not receive any anti-neoplastic treatment. However, after adjusting for treatment modalities, almost the same prognostic factors were identified (data not shown). Although the survival model has been internally validated, the lack of external validation needs to be further studied to confirm the predictive accuracy and benefit of the nomogram.

\section{REFERENCES}

Bajorin DF, Dodd PM, Mazumdar M, Fazzari M, McCaffrey JA, Scher HI, Herr H, Higgins G, Boyle MG (1999) Long-term survival in metastatic transitional-cell carcinoma and prognostic factors predicting outcome of therapy. J Clin Oncol 17(10): 3173-3181

Bellmunt J, Choueiri TK, Fougeray R, Schutz FA, Salhi Y, Winquist E, Culine S, von der Maase H, Vaughn DJ, Rosenberg JE (2010) Prognostic

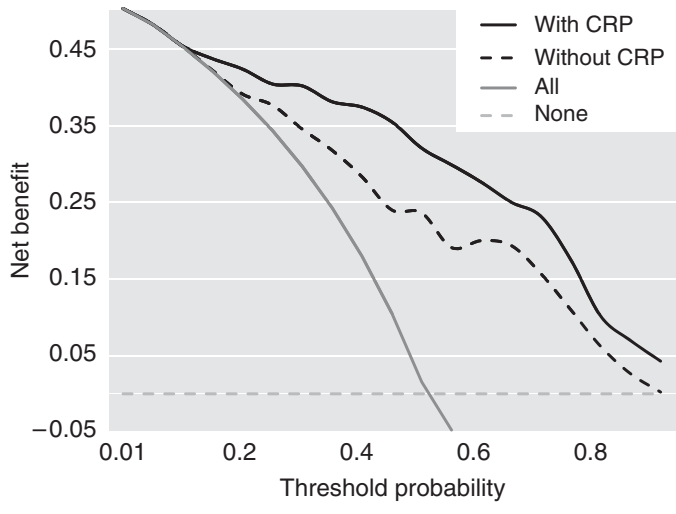

Figure 5 Decision curve analyses for overall survival predictions. The dashed line indicates the net benefit of using the model without CRP, and the solid line indicates the net benefit of using the model with CRP. The assumptions that all patients will be alive and that no patients will be dead are represented by grey and black lines, respectively. Abbreviation: $\mathrm{CRP}=\mathrm{C}$-reactive protein.

In conclusion, we demonstrated the prognostic impact of CRP that was incorporated into a nomogram and resulted in improved predictive accuracy for patients with advanced UC.

\section{Conflict of interest}

The authors declare no conflict of interest.

factors in patients with advanced transitional cell carcinoma of the urothelial tract experiencing treatment failure with platinum-containing regimens. J Clin Oncol 28(11): 1850-1855

Chen CH, George SL (1985) The bootstrap and identification of prognostic factors via Cox's proportional hazards regression model. Stat Med 4(1): $39-46$ 
Gakis G, Todenhoefer T, Renninger M, Schilling D, Sievert KD, Schwentner C, Stenzl A (2011) Development of a new outcome prediction model in carcinoma invading the bladder based on preoperative serum C-reactive protein and standard pathological risk factors: the TNR-C score. BJU Int 108(11): 1800-1805

Harrell Jr FE, Califf RM, Pryor DB, Lee KL, Rosati RA (1982) Evaluating the yield of medical tests. JAMA 247(18): 2543-2546

Iimura Y, Saito K, Fujii Y, Kumagai J, Kawakami S, Komai Y, Yonese J, Fukui I, Kihara K (2009) Development and external validation of a new outcome prediction model for patients with clear cell renal cell carcinoma treated with nephrectomy based on preoperative serum C-reactive protein and TNM classification: the TNM-C score. J Urol 181(3): 1004-1012

Ingram DG, Kattan MW (2011) Risk grouping versus risk continuum in patients with clinically localized prostate cancer: a taxometric test. J Urol 184(5): 1937-1941

Jessen C, Agerbaek M, Von Der Maase H (2009) Predictive factors for response and prognostic factors for long-term survival in consecutive, single institution patients with locally advanced and/or metastatic transitional cell carcinoma following cisplatin-based chemotherapy. Acta Oncol 48(3): 411-417

Karakiewicz PI, Hutterer GC, Trinh QD, Jeldres C, Perrotte P, Gallina A, Tostain J, Patard JJ (2007) C-reactive protein is an informative predictor of renal cell carcinoma-specific mortality: a European study of 313 patients. Cancer 110(6): 1241-1247

Lughezzani G, Budäus L, Isbarn H, Sun M, Perrotte P, Haese A, Chun FK, Schlomm T, Steuber T, Heinzer H, Huland H, Montorsi F, Graefen M, Karakiewicz PI (2010) Head-to-head comparison of the three most commonly used preoperative models for prediction of biochemical recurrence after radical prostatectomy. Eur Urol 57(4): 562-568

López-Novoa JM, Nieto MA (2009) Inflammation and EMT: an alliance towards organ fibrosis and cancer progression. EMBO Mol Med 1(6-7): 303-314

Malmström PU (2008) Why has the survival of patients with bladder cancer not improved? BJU Int 101(3): 267-269

Ramsey S, Lamb GW, Aitchison M, Graham J, McMillan DC (2007) Evaluation of an inflammation-based prognostic score in patients with metastatic renal cancer. Cancer 109: 205-212
Roxburgh CS, McMillan DC (2010) Role of systemic inflammatory response in predicting survival with primary operable cancer. Future Oncol 6(1): $149-163$

Saito K, Kawakami S, Ohtsuka Y, Fujii Y, Masuda H, Kumagai J, Kobayashi T, Kageyama Y, Kihara K (2007) The impact of preoperative serum C-reactive protein on the prognosis of patients with upper urinary tract urothelial carcinoma treated surgically. BJU Int 100(2): 269-273

Saito K, Kihara K (2011) C-reactive protein as a biomarker for urological cancers. Nat Rev Urol 8(12): 659-666

Sakura M, Kawakami K, Ishioka J, Fujii Y, Yamamoto S, Iwai A, Numao N, Saito K, Koga F, Masuda H, Kumagai J, Yonese J, Fukui I, Kihara K (2011) A novel repeat biopsy nomogram based on three dimensional extended biopsy. Urology 77(4): 915-920

Shariat SF, Karakiewicz PI, Suardi N, Kattan MW (2008) Comparison of nomograms with other methods for predicting outcomes in prostate cancer: A critical analysis of the literature. Clin Cancer Res 14(14): 4400-4407

Tatokoro M, Saito K, Iimura Y, Fujii Y, Kawakami S, Kihara K (2008) Prognostic impact of postoperative C-reactive protein level in patients with metastatic renal cell carcinoma undergoing cytoreductive nephrectomy. J Urol 180(2): 515-519

Vickers AJ, Cronin AM (2010) Traditional statistical methods for evaluating prediction models are uninformative as to clinical value: towards a decision analytic framework. Semin Oncol 37(1): 31-38

Vickers AJ, Cronin AM, Elkin EB, Gonen M (2008) Extensions to decision curve analysis, a novel method for evaluating diagnostic tests, prediction models and molecular markers. BMC Med Inform Decis Mak 8: 53

Vickers AJ, Elkin EB (2006) Decision curve analysis: a novel method for evaluating prediction models. Med Decis Making 26(6): 565-574

von der Maase H, Sengelov L, Roberts JT, Ricci S, Dogliotti L, Oliver T, Moore MJ, Zimmermann A, Arning M (2005) Long-term survival results of a randomized trial comparing gemcitabine plus cisplatin, with methotrexate, vinblastine, doxorubicin, plus cisplatin in patients with bladder cancer. J Clin Oncol 23(21): 4602-4608

Yoshida S, Saito K, Koga F, Yokoyama M, Kageyama Y, Masuda H, Kobayashi T, Kawakami S, Kihara K (2008) C-reactive protein level predicts prognosis in patients with muscle-invasive bladder cancer treated with chemoradiotherapy. BJU Int 101(8): 978-981

This work is published under the standard license to publish agreement. After 12 months the work will become freely available and the license terms will switch to a Creative Commons Attribution-NonCommercial-Share Alike 3.0 Unported License. 